\title{
The Study on Problems and Their Answer in Elementary Education Quality Monitoring
}

\author{
Dongxing Li ${ }^{1}$, Zuying Luo ${ }^{1,2, ~ *, ~ W e n q u a n ~ C h a n g ~}{ }^{1}$ \\ ${ }^{1}$ College of Information Science and Technology, Beijing Normal University, Beijing, P. R. China \\ ${ }^{2}$ Engineering Research Center of Virtual Reality and Applications, Ministry of Education, Beijing, P. R. China
}

\section{Email address:}

lidx@bnu.edu.cn (Dongxing Li), luozy@bnu.edu.cn (Zuying Luo), changcwq@mail.bnu.edu.cn (Wenquan Chang)

${ }^{*}$ Corresponding author

\section{To cite this article:}

Dongxing Li, Zuying Luo, Wenquan Chang. The Study on Problems and Their Answer in Elementary Education Quality Monitoring. Science Innovation. Vol. 5, No. 4, 2017, pp. 220-226. doi: 10.11648/j.si.20170504.15

Received: March 30, 2017; Accepted: April 14, 2017; Published: May 11, 2017

\begin{abstract}
Elementary education quality (BEQ) monitoring plays the key role in BEQ improvement. Since BEQ monitoring employs traditional educational measurement methodology (including test, questionnaires, interview, observation, literature and so on) for information collection, today's BEQ shows its disadvantages on high human cost, small sample, poor timeliness, much more steady-state and secondary data, unable to dynamically monitor all the samples in the whole education process, paying more attentions on qualitative evaluation and less on quantitative evaluation. As the result, BEQ's credibility and public trust are harmed. Recently, education big data is limited to BEQ of online education and however, cannot be used to monitor the classroom teaching effect (CTE). Based on the latest information technology including affective computing, this work proposes a novel CTE auto-monitoring model and develops an initial prototype system named as CAISBNU. Initial studies draw the exciting conclusion. This system leads to much more advantages on high automation and efficiency, perfect timeliness, easy integration, low operation cost, generating education big data for deep researches on basic education, which can make some contribution for education of China.
\end{abstract}

Keywords: Educational Measurement, Education Monitoring, Classroom Teaching, Instruction Evaluation, Education Big Data, Educational Technology, Affective Computing

\section{中国基础教育质量监测所面临的问题及其解决方法的研究}

\author{
李东兴 ${ }^{1}$, 骆祖莹 ${ }^{1,2 *}$, 常文泉 ${ }^{1}$ \\ '信息科学与技术学院, 北京师范大学, 北京, 中国 \\ 虚拟现实应用教育部工程研究中心, 教育部, 北京, 中国
}

邮箱

lidx@bnu.edu.cn（李东兴）, luozy@bnu.edu.cn（骆祖莹）, changcwq@mail.bnu.edu.cn（常文泉）

摘要: 基础教育质量监测是基础教育质量提升的关键。现行的基础教育质量监测采用测验、问卷、访谈、观察、文献 等传统技术手段进行信息采集，存在高人力成本、采样量小、时效性差、静态/间接数据过多、无法进行全样本全过程 动态监测、重视定性评价/忽视定量评价等诸多问题, 损害了基础教育监测结果的可信度与公信力。目前教育大数据只 能对在线学习效果进行监测研究, 难以对课堂教学效果实施监测。基于情感计算等信息技术, 本文提出了一种全新的 课堂教学效果自动监测模型, 并开发了 “凯撒百努” 课堂教学效果自动监测原型系统。初步的研究结果表明: 该系统 具有自动高效、时效性好、易于集成、运行成本低、可以产出面向基础教育深层研究的教育大数据等诸多技术优势, 对推动中国教育的健康发展有一定的贡献。 
关键词: 教育测量, 教育监测, 课堂教学, 教学评估, 教育大数据, 教育技术, 情感计算

\section{1. 引言}

中小学教育是中国基础教育的主体。在学校中, 学生 不仅在课堂上学到了知识, 而且通过与同学、老师、学校 的面对面互动, 培养了情商。鉴于在线教育只能提供知识 教育, 无法提供情商教育, 在线教育只能是学校教育的补 充手段, 无法撼动正规的学校课堂教育的主流地位。因此, 提高基础教育质量的关键在于提高课堂教学的效果。如何 对课堂教学效果进行有效测量具有重要的研究意义 $[1,2,3]$, 是教育测量、教育评价、教育评估、教育监测、 教育监控的主要研究内容 $[4]$ 。

课堂教学效果监测主要采用测验、问卷、访谈、观察、 文献、档案袋等经典的技术手段进行信息采集 $[4,5]$, 存 在高人力成本、采样量小、时效性差、静态/间接数据过 多、无法进行全样本全过程动态监测、重视定性评价/忽 视定量评价等诸多问题, 损害了基础教育监测结果的可信 度与公信力 $[6]$ 。

近年来，随着信息技术的高速发展，在高等教育和职 业教育领域, 涌现出了网络学校、MOOC等在线教育模式。 通过学生在网上所留下的数字痕迹可以积累出教育大数 据, 通过数据挖掘技术可以对教学效果进行定量测量 [7-8]。通过摄像头对学生的脸部表情进行识别, 还可以 对学生的学习效果进行实时测量 [9]。

鉴于手机等移动设备在课堂教学中的应用争议较大 [10], 采用移动设备上的摄像头对学生脸部表情进行近距 离摄像不具备可行性。而先采用高清摄像头对课堂教学进 行全场景录像, 再对场景内众多学生的表情进行识别则存 在较高的技术困难, 所以目前还没有出现课堂教学效果的 自动监测系统。因此, 基础教育的大数据主要来源于考试、 问卷所获得的静态数据 $[3,11,12,13,14]$, 无法对课堂教 学效果进行全时段的动态监控。

为了满足基础教育对于课堂教学效果自动评测需要, 基于并行计算与情感计算等最新的信息技术, 本文提出了 一种全新的课堂教学效果自动监测模型, 包括高清课堂教 学视频的更新/上传/在线播放、用户注册、基于家长评价 文本情感识别的课堂教学效果校外自动监测、基于学生听 课姿态与表情识别的课堂教学效果校内自动监测等功能 模块。基于此模型, 我们开发了 “凯撒百努 (CAISBNU)” 课堂教学效果自动监测原型系统。初步的研究成果表明: 该系统有助于加强家校互动、获取家长评价信息、分析学 生心理、易于集成、能够获取构建教育大数据的结构化数 据、易于进行数据挖掘等诸多技术优势, 不仅能够很好地 满足 “家校通、校校通” 的需要 [21], 也是 “管办评分离” 急需的教育质量评估关键技术 [22]。

\section{2. 教育大数据时代的基础教育质量监测的不足}

教育大数据可以有效提高教育质量 $[16]$ 。在基础教育 的教学过程中, 采用教育测量技术可以收集到大量的数据,
如果能够对全对象（学生、教师、家长）全时段进行不间 断的数据采集, 就可以获得面向基础教育的教育大数据 $[6-8,14-17]$ 。对大数据可以采用分组比较、数据可视化、 数据挖掘、机器学习、预测等技术手段进行数据分析 [8], 服务于教育实践和教育研究。教育大数据除了可用于教育 质量监测 $[11,12,17]$ 以外, 还可用于学生减负 [14]、个性 化学习与课程分析 [14-16]、辍学行为与助学需求的预测 [15]、个性化学习、为教育决策和教育改革提供参考 [17] 等。

但从教育大数据的角度来看, 目前的基础教育质量监 测还存在如下不足之处, 必须研究全新的教育测量方法。

\section{1. 过度依赖静态数据}

过度依赖静态数据, 缺少对教学全过程监测的动态数 据, 是基础教育监测分析面临的首要问题。由于移动智能 设备用于课堂教学的争议比较大 [10], 当前基础教育的课 堂教学无法留下学生的数字痕迹, 因此无法对课堂教学进 行动态监测。事实上, 目前基础教育质量监测还是采用测 验、问卷、访谈、观察、文献、档案袋等传统的技术手段 来获得监测信息, 所获得的信息都是静态数据, 而且是间 接数据。

\section{2. 人力成本高}

人力成本过高, 导致难于进行长周期常态化监测。总 体而言, 访谈、观察、文献、档案袋等经典测量方法都需 要大量的人员进行数据采集与数据分析, 测试法所需的标 准化试卷编制需要高水平专家的大量劳动, 而问卷法不仅 需要高水平专家进行设计, 而且需要大量人手进行发放与 收集。因此, 所有的教育监测活动均需要动员、培训、实 施、处理、反馈等多个环节 [4], 只能获得采样时段的离 散信息，无法保证检测过程的连续性。

\section{3. 难以进行全样本的常态监测}

总体而言, 只有测试法能够做到全样本的全员测试, 但频繁的测试会增加学生的负担 $[5,14]$, 在减负的大背景 下, 难于使用测试法对全样本进行常态监测。

\section{4. 监测的时效性差}

由于无法进行常态监测, 目前基础教育质量监测只能 对静态的监测数据进行分析, 同时访谈、观察、文献、档 案袋等方法所获得的结果难于使用计算机进行自动处理 $[4,5]$, 所以基础教育质量监测结果的时效性比较差。

\section{5. 监测结果的公正性难以保证}

根据有限样本的时效性比较差的静态与间接数据, 目 前基础教育质量监测结果的公正性难于保证, 损害了它的 公信力 $[5,22]$ 。 


\section{3. 教育大数据在教育质量监测领域的应用现状 与所遇到的问题}

尽管教育大数据技术目前热火朝天 $[7,8,13-17]$, 但 它在基础教育质量监测领域还没有令人信服的应用, 其原 因主要在于以下几点:

\section{1. 基础教育无法进行在线教育}

目前教育大数据主要来自在线学习的数字痕迹, 而课 堂教学是基础教育的主要载体, 不仅难于进行在线教育, 而且获取学生学习痕迹的移动智能设备的使用也饱受争 议 [10], 这主要由于手机或平板电脑必须联网才能使用, 知识性学习内容的枯燥决定了自制力不强的中小学生不 能专心学习, 而会转向网络上提供的高诱惑力内容 (如电 子游戏）。

\section{2. 比较高的技术门槛}

目前MOOC等在线教育主要通过日志文件来获取学习 者汶览学习资源的数字痕迹, 日志文件数据量大、但包含 的有用信息密度低, 由日志文件构成的教育大数据使用门 槛比较高, 必须使用分组比较、数据可视化、数据挖掘、 机器学习、预测等技术手段进行数据分析 [8], 因此需要 大量的大数据人才才能开展教育大数据研究 $[15]$ 。

\section{3. 隐私和伦理的问题}

大数据时代的个性化服务是建立在对行为的追踪和 分析的基础上的, 这意味着会在某种程度上触及到个人无 意公之于众的隐私信息。此类信息的所有权和使用权的归 属并不明确, 数据可能遭到滥用 [15]。因此, 即使能够累 积出教育大数据, 也不能随便授权给相关的技术公司使用。

\section{4. 人才缺乏}

教育大数据是教育与 IT的交叉技术, 缺少两方面都理 解的复合型人才是教育大数据应用于基础教育质量监测 所面临的大问题。由于IT技术发展日新月异，目前教育技 术专业的学者尽管都是信息技术出身, 但他们还是无法将 信息技术在大规模并行计算、情感计算、计算机视觉处理、 云计算、大数据等最新发展应用于教育研究中。

\section{4. 基于计算机辅助情感计算的课堂教学效果自 动监测模型}

针对基础教育质量监测需要全样本、全时段的动态监 测数据, 以构建易于使用的教育大数据, 我们采用最新的 大规模并行计算、情感计算、计算机视觉、计算机网络等 IT技术对课堂教学效果自动监测进行了研究, 提出了一种 可行的课堂教学效果自动监测系统模型, 并构建了一个初
步的原型系统。课堂教学效果自动监测系统的工作原理如 图1所示, 系统主要由如下功能模块构成。

\section{1. 视频采集}

先通过高清摄像头对课堂中的学生听课效果进行录 像, 存在课堂教学计算机中, 然后在课间通过网络存到课 堂教学视频库中。

\section{2. 视频库管理}

任课教师和学校网管人员对高清视频进行审查, 将合 格的视频放到在线播放列表中。学校网管人员定期对视频 库和在线播放列表进行更新。

\section{3. 用户注册与登录}

根据学生的学号, 建立用户注册与登录系统, 以保护 学生的隐私, 同时以注册表的形式、对学生及其家庭的信 息进行采集, 用于调查学生素质与学业发展的影响因素。

\section{4. 在线播放}

家长登录网站后, 可以从在线播放列表中选取感兴趣 的高清视频进行在线观看。为了保护学生的隐私, 本系统 不提供在线下载的功能。

\section{5. 家长反馈评价意见的文本输入}

系统提供一个最大长度为 130 个字的文本输入窗口, 以收集家长对于学生听课效果的反馈意见。

\section{6. 家长评价意见的文本情感识别}

采用文本情感识别技术对家长的反馈意见进行文本 情感计算, 以获取课堂教学效果的家长评价值。

\section{7. 自然表情的并行识别}

采用大规模并行计算技术，对高清视频中的众学生人 脸进行并行的人脸检测、人脸识别、自然表情的并行识别, 最后获得每个时刻每名学生听课表情的愉悦度和激活度 测量值。

\section{8. 基于自然表情的学习心理绑定}

根据学生的自然表情的愉悦度和激活度, 从教育与心 理专家的先验知识出发, 对学生表情所对应的学习心理进 行绑定, 并采用家长的评价值对整个绑定过程进行整体校 准。

\section{9. 基于学生表情的听课效果识别}

对标定出来的学生学习心理的序列 (基于时间轴) 进 行统计与分类, 获取每个学生的听课效果。对全班学生的 听课效果进行统计, 可以获得该次授课的教学效果。 


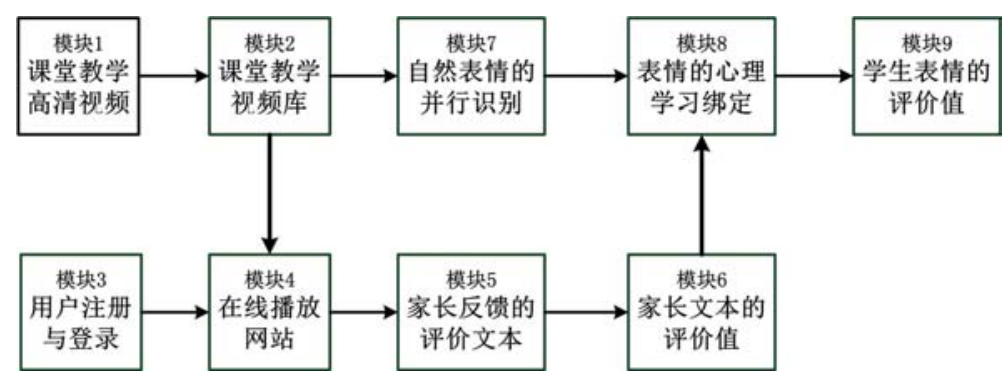

图1 课堂教学效果自动监测原型系统的工作原理。

5. “凯撒百努” 课堂教学效果自动监测原型系 统

经过一年多的研究, 包括文献调研和初步实验, 在视 频采集与网站构建、家长评价文本的输入与文本情感识别、 人脸检测/识别/表情识别等三个领域取得了初步的研究 成果, 具体的研究成果被集成在 “凯撒百努 (CAISBNU) ” 课堂教学效果自动监测原型系统中, 其中CAISBNU为英文
名 “Computer Aided Instruction System of Beijing Normal University” 的首字母缩写。

如图2所示, “凯撒百努” 课堂教学效果自动监测原 型系统的课堂教学高清视频在线播放网站的登录界面, 为 了保障学生的个人隐私, 网站只对在校学生的家长与教师 开放, 家长的登录号为学生的学号, 教师的登录号为其工 作证号。

\section{计算机辅助教育系统 CAISBNU}

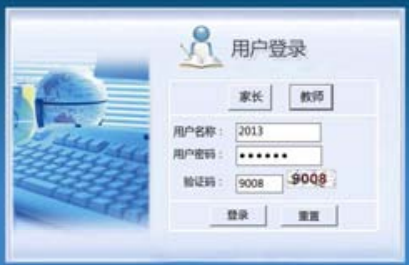

图2 课堂教学高清视频在线播放网站的登录网页。

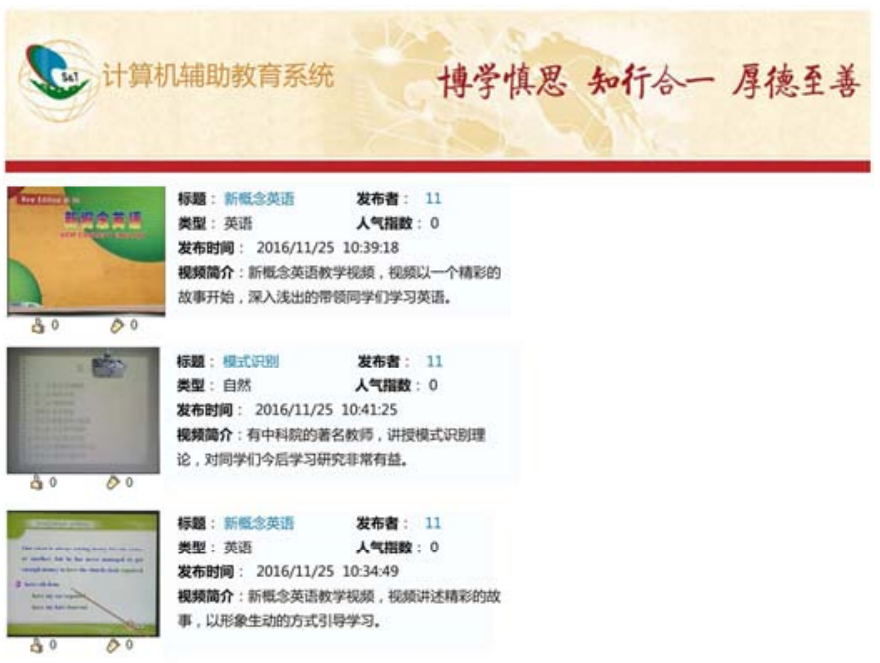

图3 课堂教学高清视频的在线播放列表的截图。 
如图3所示, 课堂教学高清视频通过播放列表给出, 通过上传来增添在线播放的文件。点击后视频文件后, 在 线播放, 如图4所示, 我们开发的在线播放页面不仅提供
全部的视频播放功能, 而且还给出了对于此视频的其他家 长评价, 以及供家长输入评价意见的文本输入窗口。

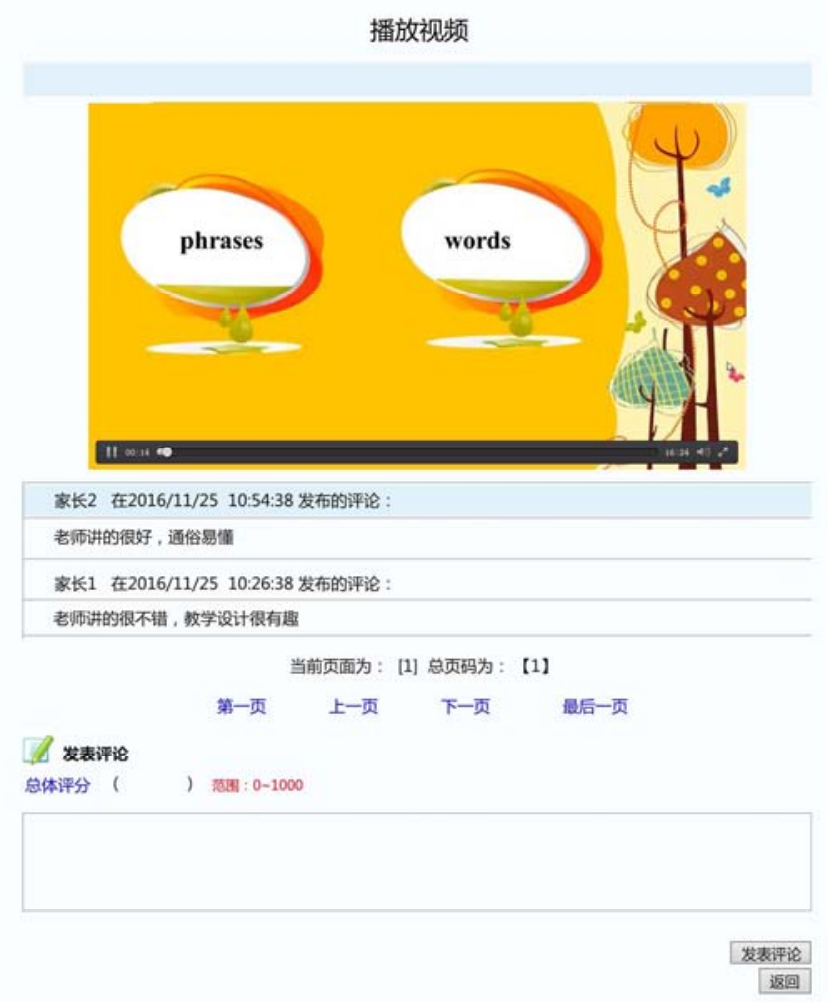

图4 在线播放与评价文本输入的截图。

\section{6. 基础教育课堂教学效果自动监控的技术优势}

与现有教育质量监测所采用的教育测量传统方法相 比, 基于最新的信息技术所开发的课堂教学效果自动监测 系统具有如下明显的技术优势。

\section{1. 时效性好}

由于可以当天对所有课堂教学视频进行处理, 根据所 有学生的听课表情进行听课效果的自动评测, 以获得该次 课堂教学效果的监测数据序列（沿时间轴展开）, 所以课 堂教学效果自动监测系统可以对全班学生的整个课堂教 学期间听课表情进行动态监测, 而且可以当天就获得监测 数据, 所以时效性非常好。

\section{2. 提供泛在的监测手段}

由于课堂教学视频必须在线播放, 所以课堂教学效果 自动监测系统必须构建一个网站, 目前信息化工作进展好 的示范学校已经建立了学校网站 [19], 只要将课堂教学效 果监测系统的网页链接到学校网站上即可; 而对于没有条 件的普通学校, 则可以由教育主管部门委托专业公司在教 育云上构建各自的教学网站 [20], 进行在线播放。这样只
要能够上网, 用户就能够通过手机等智能移动终端对课堂 教学过程进行泛在监测。

\section{3. 促进家校互通}

教师、家长、学生在交互中, 共同提高课堂教学质量。 家校互通是提高基础教育质量的重要手段 [18], 但为了保 证中小学生的人身安全和提供不受打扰的教学秩序, 目前 有围墙的学校都谢绝外人 (包括家长) 入内, 家长当然就 更不能随便进入教室, 这就造成了课堂教学的黑盒现象。 提供在线视频播放, 可以将课堂教学变为白盒过程, 家长 不仅可以根据自己孩子的课堂听课表现, 来共同研究提高 课堂听课效果的方法, 还可以通过留言板将自己对课堂教 学评价及其改进意见发到线上, 群策群力地帮助教师改进 教学方法、提高教学效果。

\section{4. 提高教学效果}

将课堂教学由无监督过程变为有监督过程, 提高教学 效果。对于课堂中的当事人一学生与教师而言, 由于课堂 教学变成了受教育主管部门和广大家长监督的过程, 他们 就会有意识地改进自己的角色, 同时教育主管部门的改进 意见和广大家长的建议也会帮助他们进一步完善自己的 角色。 


\section{5. 易于集成}

便于构建全国基础教育质量监测网络，提供实时的监 测数据与视频。可以对所有学校的课堂教学效果监测系统 网页进行集成 [21], 构建全国性的基础教育质量监测网络, 教育主管部门可以随时随地对任意学校的课堂教学进行 突击检查, 降低监测成本。

\section{6. 数据易于利用}

提供课堂教学质量评价的结构性数据, 其构建的教育 大数据易于利用。与MOOC等在线教育技术积累网络日志所 成的教育大数据 $[7-8]$ 相比, 课堂教学效果自动监测系统 提供了三类教育大数据: (A) 课堂教学效果的家长评价值、 （B）基于学生表情的评价值、(C) 用户注册所提供的学生背 景材料, 均为可以直接利用的数据, 同时节约存储空间。 不仅可以将 (A) 和 (B) 的评价值与学生的学业进行相关研 究, 而且可以将学生的学业数据与学生的背景进行相关研 究。因此, 降低了教育大数据的收集与利用的难度。

\section{7. 为学生与教师减负}

课堂教学效果自动监测能够提供教学效果的评价值, 可以替代大部分用于测试学习效果的考试, 达到为学生和 教师减负的目的 $[14]$ 。

\section{8. 有效提高基础教育质量监测的公正性, 增强其公信 力}

课堂教学效果自动监测系统提供的数据都是客观的 数据, 能够保证监测的客观性。基于该系统构建的全国基 础教育质量监测网络可以根据监测数据、进行客观公正的 抽样, 再聘用相关经验丰富的专家对这些小样本进行高耗 时的访谈与观察, 以获取更为直观、深入、细节的教育评 价信息。

\section{9. 保持课堂教学过程的完整性}

课堂教学效果自动监测系统只对课堂教学效果进行 自动监测, 在课堂教学过程中, 监测系统是一个默默无声 的旁观者, 不对教师的教和学生的学产生直接作用, 因此, 它是一种间接的计算机辅助教育技术, 保持了课堂教学过 程的完整性。这就最大限度地降低了教学主体 (教师和学 生) 的抵触情绪, 有利于课堂教学效果自动监测系统的推 广应用。

总之, 课堂教学效果自动监测系统也可以通过提供课 堂教学效果的评价数据, 从而大幅度提高基础教育质量。

\section{7. 课堂教学效果自动监控系统研发与应用对于 中国教育的重要意义}

基于以上所列的课堂教学效果自动监控的技术优势, 课堂教学效果自动监测系统的研发与应用可以有效推动 中国教育的健康发展, 其作用主要体现在如下方面。

\section{1. 全面推动基础教育质量的提升}

课堂教学效果自动监控可以很好地满足家校通、校校 通的需求, 其提供的课堂教学效果监测数据正是 “管办评 分离” 所需客观公正的第三方评测结果, 因此能够全面推 动基础教育质量的提升。

\section{2. 全面推动教育科学的发展}

相比于日新月异的信息技术发展, 教育科学的发展相 对是比较缓慢的, 其所依赖的实验数据还是采用测验、问 卷、访谈、观察、文献、档案袋等传统技术手段所获得的 小容量数据, 无法利用最新的教育大数据技术来揭示深层 次的教育规律。课堂教学效果自动监控所提供的教育大数 据能够将教育科学带入一个快速的全新发展阶段。

\section{3. 全面推动教育技术的发展}

教育技术研究人员将信息技术引入教育领域, 对教学 方法、手段、内容进行改进。随着信息技术的进步，远程 教育、在线教育、移动教育、虚拟教育、MOOC等教育技术 不断普及, 然而都需要直接对课堂教学过程进行改动, 教 师需要付出额外的工作量, 所以教师会产生抵触情绪, 这 些技术都没有在基础教育行业中得到大规模应用。相反, 课堂教学效果自动监测是一项间接的教育技术, 不需要教 师需要付出额外的工作量, 还可以为教师改进教学提供大 量的课堂教学效果实测数据, 因此具有大规模应用的有利 条件。

\section{4. 推动师范院校的心理科学发展}

课堂教学效果自动监测需要依据学生的面部表情和 坐姿来判断学生的学习心理, 能够推动师范院校的心理科 学发展。

\section{5. 全面推动教育产业的发展}

由于缺乏足够的教学软件支持, 并增加教师的工作量, 现有的电子白板、电子书包等教育技术都没有带来教育产 业的大发展。课堂教学效果自动监测不仅需要在教室中增 加高清摄像系统, 而且需要构建在线教学视频播放系统, 同时需要高性能计算系统来对教学视频进行众多学生的 表情识别和学习心理识别, 其产生的教学大数据也需要集 成到集成教育质量监测的云中心里进行大数据分析。因此, 课堂教学效果自动监测能够全面带动教育产业的发展。

\section{6. 全面推动师范院校的信息学科发展}

由于缺乏行业背景, 师范院校发展计算机、自动化、 电子、微电子、软件等信息学科都遇到了需求不足的问题。 课堂教学效果自动监测可以为师范院校的计算机、自动化、 电子、软件学科发展提供强烈的行业需求, 因此能够全面 推动师范院校的信息学科发展。 


\section{8. 结论}

本文不仅从教育大数据的角度对基础教育质量监测 的不足进行了深入的论述, 而且也指出了现有教育大数据 研究主要集中于在线教育, 无法满足基础教育质量监测采 集的需求。基于情感计算等最新的信息技术发展, 本文提 出了一种全新的基础教育课堂教学效果自动监测模型, 并 开发了 “凯撒百努” 课堂教学效果自动监测原型系统。初 步研究结果表明: 本文提出的课堂教学效果自动监测模型 能够解决现有基础教育监测所面临的各种问题, 其获得的 教育大数据易于使用, 能够全面推动中国教育的健康发展。

鉴于我们主要从技术角度对基础教育课堂教学效果 自动监测进行研究, 可能对教育实践所出现的各种问题不 够了解, 为了抛砖引玉, 我们仅根据初步的研究成果就将 我们的研究工作进行报道, 希望教育界的专家多提宝贵的 建议, 以开发出满足基础教育质量监测要求的课堂教学自 动监测系统。

\section{致谢}

本文工作获得国家自然科学基金 (61274033) 和中央 高校基本科研业务费专项资金项目2014KJJCA15的联合资 助。

\section{参考文献}

[1] 辛涛, 田伟, 邹舟. 教育结果公平的测量及其对基础教育 发展的启示 $[J]$. 清华大学教育研究, 2010, 31 (2) : 4-5。

[2] 杨涛, 辛涛, 董奇. 法国基础教育质量测评体系探析 [J]. 比 较教育研究, 2013 (4) : 60-65。

[3] 滕珺, 朱晓玲. 大数据在美国基础教育中的运用 $[\mathrm{J}]$. 人民 教育, 2014(1):74-76。

[4] 金娣, 王钢. 教育评价与测量 [M]. 教育科学出版社, 2007。

[5] 徐勇. 大数据时代观下的教育质量监控 (上) $[\mathrm{J}]$. 师道, 2014 (4)。
[6] 卢立涛, 梁威, 沈茜. 我国课堂教学评价现状反思与改进 路径 $[J]$ 。中国教育学刊, 2012(6) :43-47。

[7] 魏顺平. 学习分析技术: 挖掘大数据时代下教育数据的价 值 [J]。现代教育技术，2013, $23(2): 5-11$ 。

[8] 张羽, 李越. 基于MOOCs 大数据的学习分析和教育测量介绍 $[J]$. 清华大学教育研究, 2013, 34 (4) :22-26。

[9] 李勇帆, 李里程. 情感计算在网络远程教育系统中的应用: 功能、研究现状及关键问题* $[\mathrm{J}]$. 现代远程教育研究, 2013 (2) : 100-106。

[10] 张虹. 推进手机移动学习: 中小学教师态度与需求 $[\mathrm{J}]$. 现 代远程教育研究, 2012(5):71-78。

[11] 徐勇. 大数据时代观下的教育质量监控 (下) $[\mathrm{J}]$. 师道 - 情 智, 2014(5) : 31-33。

[12] 刘晓庆. 大规模学业评价研究 [D]. 华中师范大学, 2013。

[13] 徐克强, 陈秋蓉. 基础教育质量监测与数据库建设探析 [J]．中国教育信息化，2011(24):25-28。

[14] 王震一. 教育大数据或可破解 “减负” 难题 $[\mathrm{J}]$. 中小学信 息技术教育，2013(7): 14-15。

[15] 张燕南, 胡继岳. 关于大数据应用于教育的思考 $[\mathrm{J}]$. 中国 电力教育, $2013,2013(32): 5-7$ 。

[16] 何克抗. 大数据面面观 $[J]$. 电化教育研究, 2014 (10) : 8-16。

[17] 㚞平军. 家校互动: 形式、内容及现实思考 $[\mathrm{J}]$ 。基础教育 研究, 2010 (11) : 8-10。

［18］李芒，逯行． “集聚方效应”下基础教育信息化发展：思 路、模式和效果 $*-$ - 以北京市海淀区基础教育信息化为例 $[\mathrm{J}]$ ．中国电化教育，2014(10):7-15。

[19] 王康，廖汉文，张岩．云计算在基础教育信息化建设中的 应用研究 $[J]$ 。现代教育技术，2012, 22(4) : 104-106。

[20］祝智庭，管玨琪。我国基础教育信息化新发展：从 “班班 通” 到 “教育云” [J]。中国教育信息化, 2011 (14) : 4-8。

[21] 杨志刚. 基础教育管办评分离的实践探索与理论分析 [J]. 中国教育学刊, 2014(7):7-9。 